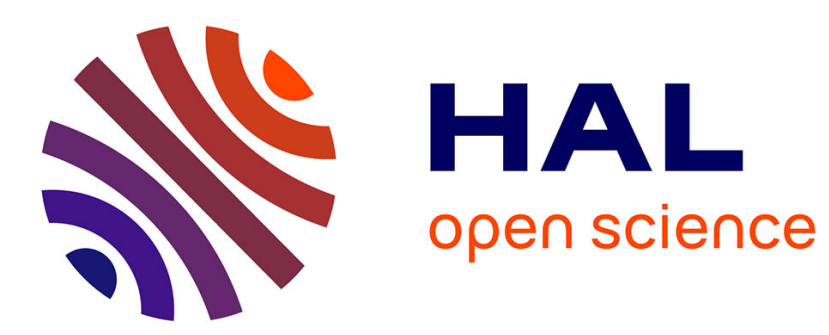

\title{
Mesures de stabilité instantanée de l'énergie de l'accélérateur
}

\author{
J. Gerber, J.P. Vivien, A. Macher, R. Armbruster
}

\section{To cite this version:}

J. Gerber, J.P. Vivien, A. Macher, R. Armbruster. Mesures de stabilité instantanée de l'énergie de l'accélérateur. Revue de Physique Appliquée, 1969, 4 (2), pp.167-168. 10.1051/rphysap:0196900402016700 . jpa-00243199

\section{HAL Id: jpa-00243199 https://hal.science/jpa-00243199}

Submitted on 1 Jan 1969

HAL is a multi-disciplinary open access archive for the deposit and dissemination of scientific research documents, whether they are published or not. The documents may come from teaching and research institutions in France or abroad, or from public or private research centers.
L'archive ouverte pluridisciplinaire HAL, est destinée au dépôt et à la diffusion de documents scientifiques de niveau recherche, publiés ou non, émanant des établissements d'enseignement et de recherche français ou étrangers, des laboratoires publics ou privés. 


\title{
MESURES DE STABILITÉ INSTANTANÉE DE L'ÉNERGIE DE L’AGGÉLÉRATEUR
}

\author{
J. GERBER, J. P. VIVIEN, A. MACHER et R. ARMBRUSTER, \\ Institut de Recherches Nucléaires, Strasbourg-Cronenbourg.
}

\begin{abstract}
Résumé. - Des figures de Lissajous permettent de visualiser la pulsation hyperfréquence d'un faisceau de particules monoénergétiques.

Abstract. - A time-of-flight analysis using UHF electric fields giving the energy stability of a Van de Graaff beam has been made.
\end{abstract}

Le montage réalisé était destiné à l'étude d'un faisceau pulsé de particules en vue de mesurer des durées de vie dans la gamme de $10^{-10}$ à $10^{-11} \mathrm{~s}$ [1]. Celui-ci est formé de deux parties, d'une part d'un système analyseur constitué de deux cavités hyperfréquences, équivalent à un champ électrique tournant de période $400 \mathrm{ps}$, perpendiculaire au faisceau, d'autre part d'une pulsation de faisceau associant une cavité déflectrice synchrone avec l'analyseur et une fente (fig. 1).

Quand le faisceau est continu, l'analyseur fait tourner le faisceau et on visualise ainsi sur un quartz une échelle de temps de 400 ps sous forme de figure de Lissajous. Le cercle est la meilleure échelle. Le dispositif de pulsation fonctionnant, le cercle est réduit à deux tirets symétriques visualisant les paquets de particules. Leurs positions dépendent des différences de phase fixes entre les trois champs déflecteurs et surtout du temps-de-vol entre le premier champ déflecteur et la fente. Une variation de $1,6 \mathrm{keV}$ sur cette base de vol se traduit par une rotation de $10^{\circ}$. La largeur des paquets dépend fortement des qualités optiques du faisceau, du parallélisme et de la stabilité en énergie. L'instabilité se traduit également par une rotation de deux paquets.

Pour chiffrer celle-ci, nous avons fait varier par pas le courant de l'aimant d'analyse correspondant à des pas de $1 \mathrm{keV}$ d'énergie sans retoucher à d'autres contrôles de l'accélérateur VdG $4 \mathrm{MeV}$. Cela se traduit par une rotation sur la figure de Lissajous. Les rotations

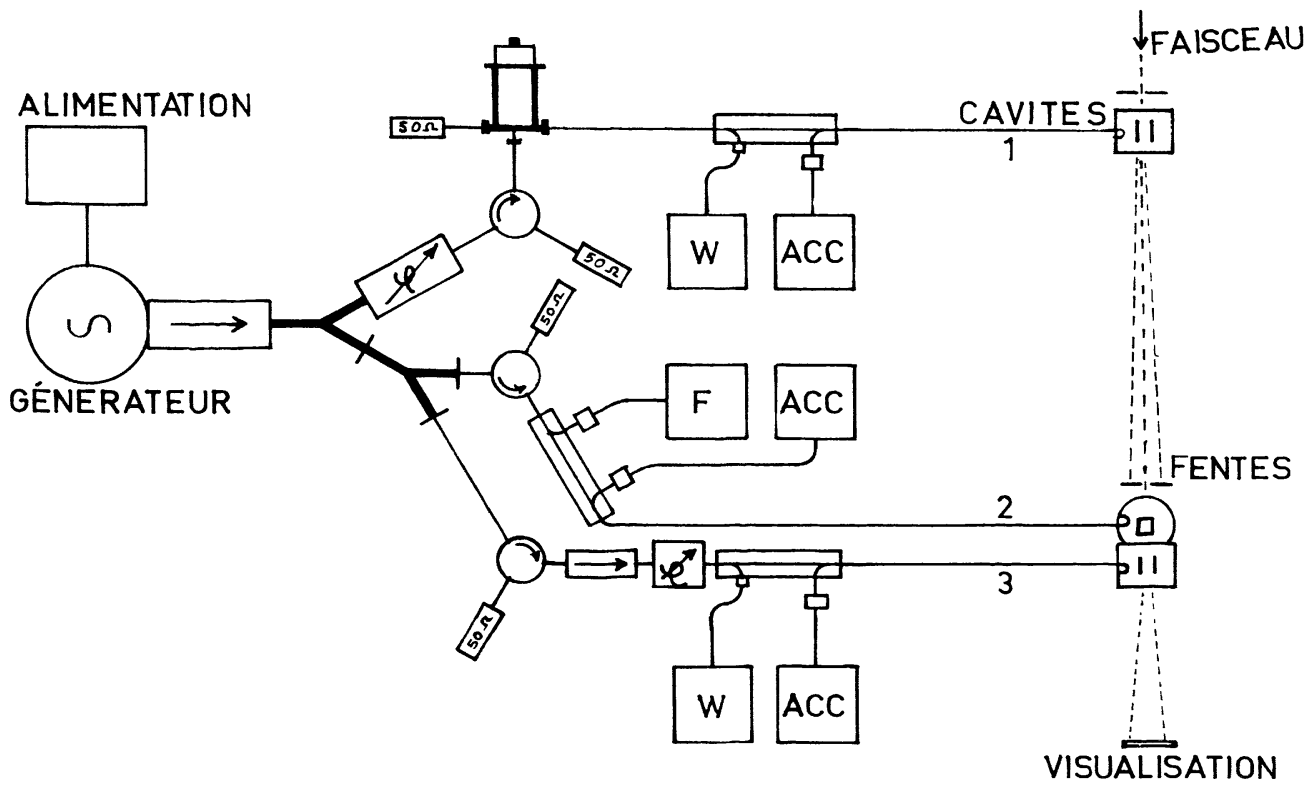

FIG. 1. - Pulsation et analyse synchrones de faisceau de particules par des champs électriques hyperfréquences. 


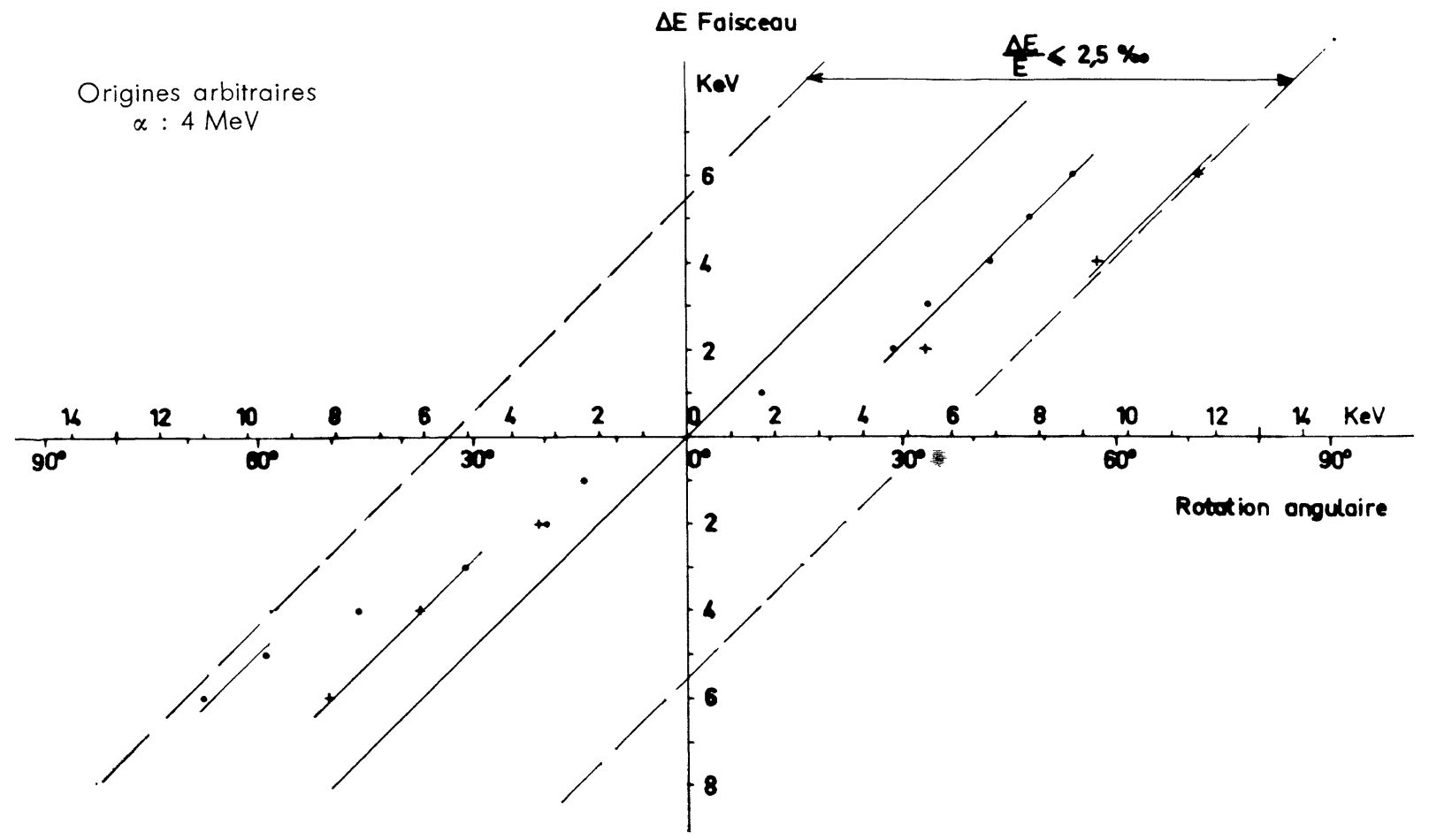

FIG. 2.

d'une série de mesures s'étalant sur une heure sont reportées sur la figure 2. L'échelle en énergie correspondante est également reportée en abscisse. En ordonnée, $\Delta E$ faisceau représente les pas d'énergie effectués sur le courant de l'aimant d'analyse de l'accélérateur. Une série de rotations montre une stabilité meilleure que $4 \times 10^{-4}$, alors que l'ensemble des points s'inscrit dans une bande correspondant à une stabilité de $2,5 \times 10^{-3}$. L'accélérateur était dans des conditions normales de fonctionnement.

\section{BIBLIOGRAPHIE}

[1] Gerber (J.) et al., ce colloque. 